\title{
Distribution Locational Marginal Pricing for Optimal Electric Vehicle Charging through Chance Constrained Mixed-Integer Programming
}

\author{
Liu, Zhaoxi; Wu, Qiuwei; Oren, Shmuel S.; Huang, Shaojun; Li, Ruoyang; Cheng, Lin
}

Published in:

IEEE Transactions on Smart Grid

Link to article, DOI:

10.1109/TSG.2016.2559579

Publication date:

2018

Document Version

Peer reviewed version

Link back to DTU Orbit

Citation (APA):

Liu, Z., Wu, Q., Oren, S. S., Huang, S., Li, R., \& Cheng, L. (2018). Distribution Locational Marginal Pricing for Optimal Electric Vehicle Charging through Chance Constrained Mixed-Integer Programming. IEEE Transactions on Smart Grid, 9(2). https://doi.org/10.1109/TSG.2016.2559579

\section{General rights}

Copyright and moral rights for the publications made accessible in the public portal are retained by the authors and/or other copyright owners and it is a condition of accessing publications that users recognise and abide by the legal requirements associated with these rights.

- Users may download and print one copy of any publication from the public portal for the purpose of private study or research.

- You may not further distribute the material or use it for any profit-making activity or commercial gain

- You may freely distribute the URL identifying the publication in the public portal 


\title{
Distribution Locational Marginal Pricing for Optimal Electric Vehicle Charging through Chance Constrained Mixed-Integer Programming
}

\author{
Zhaoxi Liu, Qiuwei Wu, Senior Member, IEEE, Shmuel S. Oren, Fellow, IEEE, Shaojun Huang,
} Ruoyang Li and Lin Cheng

\begin{abstract}
This paper presents a distribution locational marginal pricing (DLMP) method through chance constrained mixed-integer programming designed to alleviate the possible congestion in the future distribution network with high penetration of electric vehicles (EVs). In order to represent the stochastic characteristics of the EV driving patterns, a chance constrained optimization of the EV charging is proposed and formulated through mixed-integer programming (MIP). With the chance constraints in the optimization formulations, it guarantees that the failure probability of the EV charging plan fulfilling the driving requirement is below the predetermined confidence parameter. The efficacy of the proposed approach was demonstrated by case studies using a 33-bus distribution system of the Bornholm power system and the Danish driving data. The case study results show that the DLMP method through chance constrained MIP can successfully alleviate the congestion in the distribution network due to the EV charging while keeping the failure probability of EV charging not meeting driving needs below the predefined confidence.
\end{abstract}

Index Terms-Chance constrained programming, congestion management, distribution locational marginal pricing (DLMP), distribution system operator (DSO), electric vehicle (EV).

\section{NOMENClATURE}

A. Sets:

$L$

$N$

$R_{j}$

$R_{j+}$

set of lines in the distribution network

set of buses in the distribution network

set of all possible driving pattern realizations of $\mathrm{EV} j$

subset of possible driving pattern realizations of EV $j$ with a probability over the confidence parameter $\epsilon$

This work was supported by Nordic Energy Research (Norden) through the "Nordic Power Road Map 2050: Strategic Choices towards Carbon Neutrality (NORSTRAT)” project.

Z. Liu, Q. Wu and S. Huang are with Center for Electric Power and Energy (CEE), Department of Electrical Engineering, Technical University of Denmark (DTU), 2800 Kgs. Lyngby, Denmark (e-mail: zhliu@elektro.dtu.dk; qw@ elektro.dtu.dk; shuang@ elektro.dtu.dk).

S. S. Oren and R. Li are with Department of Industrial Engineering and Operations Research (IEOR), University of California, Berkeley, CA 94704 USA (e-mail: Shmuel@berkely.edu, ruoyang@berkeley.edu).

L. Cheng is with Department of Electrical Engineering, Tsinghua University, Beijing 100084, China (e-mail: chenglin@mail.tsinghua.edu.cn).
$R_{j-}$

$T$

V

$V_{i}$

\section{B. Parameters:}

$A_{t} \in \mathbb{R}^{n} \quad$ vector of the spot prices of the buses at time

$B_{t} \in \mathbb{R}^{n \times n} \quad$ matrix of the price sensitivity coefficients of the buses at time period $t$

$D_{l} \in \mathbb{R}^{n} \quad$ power transfer distribution factor (PTDF) coefficients of line $l$ at the buses

$d_{j, t} \quad$ driving energy consumption of the $\mathrm{EV} j$ at time

$E_{j} \in \mathbb{R}^{n} \quad$ mapping vector of the $\mathrm{EV} j$ to the load bus

$e_{j}^{-} \quad$ lower limit of the EV state of charge (SOC) level

$e_{j}^{+} \quad$ upper limit of the EV SOC level

$e_{0, j} \quad$ initial SOC level of the $\mathrm{EV} j$

$f_{l} \quad$ capacity limit of line $l$

$n \quad$ cardinality of the set of buses $N$

$p_{+} \quad$ upper power limit of the EV charging

$Q_{t} \in \mathbb{R}^{n} \quad$ conventional demand at time period $t$

$s_{j, t} \quad$ charging availability indicator of the $\mathrm{EV} j$ at time period $t$

$t_{j}^{\text {dep }} \quad$ departure time of the EV's first trip of the day

$t_{j}^{a r r} \quad$ arrival time of the EV's last trip of the day

$\alpha_{t} \quad$ spot price at time period $t$

$\beta_{t} \quad$ price sensitivity coefficient at time period $t$

$\gamma_{t} \quad$ predicted price at time period $t$

$\epsilon \quad$ confidence parameter of the probabilistic constraint

C. Variables:

$x_{j, t} \quad$ charging energy of the EV $j$ at time period $t$

$x_{t} \quad$ charging energy of an EV at time period $t$

$z_{j, k} \in\{0,1\} \quad$ binary variable of the $\mathrm{EV} j$ for the realization $k$

of the possible driving patterns

$\lambda_{l, t}^{-} \quad$ dual variable for the negative flow constraint of 


$\begin{array}{ll}\lambda_{l, t}^{+} & \begin{array}{l}\text { line } l \text { at time period } t \\ \text { dual variable for the positive flow constraint of } \\ \text { line } l \text { at time period } t\end{array} \\ D L M P_{t} \in \mathbb{R}^{n} & \begin{array}{l}\text { distributional locational marginal prices } \\ \text { (DLMPs) at time period } t\end{array} \\ \rho_{t} \in \mathbb{R}^{n} & \begin{array}{l}\text { dynamic tariffs (DTs) of the buses at time } \\ \text { period } t\end{array}\end{array}$

\section{Acronyms}

$D C P \quad$ distribution congestion price

DLMPS distribution locational marginal prices

DTs dynamic tariffs

DSO distribution system operator

EVs electric vehicles

HPs heat pumps

\section{INTRODUCTION}

D UE to the increasing concern of greenhouse gas (GHG) emission and energy supply security issues, electric vehicles (EVs) have been widely promoted. A lot of efforts have been made to promote EVs and better integrate EVs into the power system [1]-[3]. At present, the EV market has been growing around the world [4].

When there is a large-scale deployment of EVs, it will have big impacts on the future power system, especially the distribution networks [5]-[7]. With a high penetration level of EVs, congestion may occur in the distribution system without proper control. The grid congestion due to the EV demand results from the uncoordinated EV charging. It can happen at both the medium voltage (MV) and low voltage (LV) levels of the distribution systems according to the previous studies [8], [9]. The distribution system operator (DSO) can handle the congestion problems within the distribution networks by either reinforcing the system through long term planning or employing market based congestion control methods [10]. Compared to other congestion management methods, market based congestion management methods can maximize social welfare while causing least discomfort to customers [11]. The locational marginal price (LMP) concept in transmission systems is extended to the distribution systems in [12]. The work in [13]-[17] develops distribution locational marginal pricing (DLMP) to handle congestion in a distribution system with distributed generators (DGs). The work in [18] employs a dynamic tariff (DT) based on the DLMP method to handle congestion in a distribution network due to the EV demand. The DT is the congestion component of the distribution locational marginal prices (DLMPs). It reflects the congestion cost in the distribution networks. With taking into account inter-temporal characteristics, the work in [19] develops an integrated DLMP approach for congestion management from the EV charging in a distribution network. The work in [20] presents a distribution congestion price (DCP) based market mechanism derived from the DLMP concept to alleviate possible distribution system congestion due to EV and Heat Pump (HP) integration. The research in [11] proposes a DLMP based method through quadratic programming to alleviate possible congestion in a distribution system with high penetration of EVs and HPs, and solve the degeneracy issue.

Previous work has used the DLMP concept for handling congestion due to EV charging in a distribution network. However, they employ a deterministic model of EV driving patterns, which does not reflect real life stochastic characteristics of EV charging determined by aggregators (or customers). Consequently, the estimation of the EV aggregators' charging behaviors would be inaccurate and result in ineffective outcomes. The optimal EV charging strategy in the day-ahead market for the aggregators under uncertainty has been studied [21]-[23]. The driving patterns are generated randomly but considered deterministically known to the aggregators in [21]. The probabilistic properties of the driving patterns for the EV aggregator charging strategy are handled in [22], [23] by pre-constructing a set of probabilistic time-varying power and energy constraints from the driving pattern samples. Although the uncertainly of the EV charging has been studied, it has not been considered in the congestion management of the distribution network. In order to address this issue, this paper presents a DLMP method through chance constrained programming to alleviate the congestion in the distribution network due to the EV charging taking into account the uncertainties of the driving patterns. In the proposed method, the DSO determines the distribution locational marginal prices (DLMPs) by minimizing the total cost of the electricity consumption in the distribution network respecting the network constraints; the $\mathrm{EV}$ aggregators are assumed to be economically rational and their objectives are to minimize their EV charging cost with charging constraints respected. A chance constrained model is proposed in this paper such that the EV aggregators can handle the uncertainties of the driving patterns in their dayahead energy plans. The stochastic features of the EV driving patterns are taken into account in both the DSO and aggregators' optimizations through probabilistic constraints guaranteeing that energy planning satisfies driving requirements of EVs at a predefined confidence level. Because the joint distribution of the driving patterns do not follow a Gaussian distribution and the stochastic variables are not independent from each other, the joint chance constrained models in the DSO and the aggregators' optimization is difficult to solve with the stochastic approaches in previous studies by addressing the convexity of the problem [24]-[26]. In order to handle the joint chance constrained models in the DSO and the aggregators' optimizations, a formulation through mixed-integer quadratic programming (MIQP) is proposed. The chance constrained optimizations of the DSO and the aggregators are formulated and solved with the MIQP models.

The paper is organized as follows. The chance constrained modeling of the optimal EV charging is presented in detail in Section III. In Section IV, the calculation of DLMPs and DTs is described along with the formulations of the DSO and aggregators' optimizations. The results of case studies are presented and discussed in Section V, followed by conclusions. 


\section{Optimal EV ChARgING WITH CHANCE CONSTRAINTS}

In order to secure energy needs for the next day's driving, EV aggregators need to plan the EV charging schedule and submit bids in the day-ahead energy market. The objective of the optimal charging of the aggregators is to minimize the charging cost to meet the EV driving requirement. As the spot prices of the electricity can be affected by the EV charging plans, an approach was proposed in [11], [27] and [28] to predict the spot price by using the spot price together with a price sensitivity term of the demand as expressed in (1). The price sensitivity coefficient $\beta$ is determined by the merit order of the power plants in the electricity market.

$$
\gamma_{t}=\alpha_{t}+\beta_{t} x_{t}
$$

Then for a single EV, the charging cost can be expressed as,

$$
\sum_{t \in T} \gamma_{t} x_{t}=\sum_{t \in T}\left(\alpha_{t} x_{t}+\beta_{t} x_{t}^{2}\right)
$$

For aggregator $i$, the optimal energy planning for EV charging can be formulated as a standard quadratic programming model.

\section{Subject to}

$$
\min _{x_{j, t}} \sum_{j \in V_{i}} \sum_{t \in T}\left[\frac{1}{2}\left(E_{j} x_{j, t}\right)^{T} B_{t}\left(E_{j} x_{j, t}\right)+A_{t}^{T} E_{j} x_{j, t}\right]
$$

$$
\begin{array}{cl}
e_{j}^{-} \leq \sum_{t^{\prime}=t_{0}}^{t}\left(x_{j, t^{\prime}}-d_{j, t^{\prime}}\right)+e_{0, j} \leq e_{j}^{+} & \forall j \in V_{i} \forall t \in T \\
x_{j, t} \leq p_{+} s_{j, t} & \forall j \in V_{i} \forall t \in T \\
x_{j, t} \geq 0 & \forall j \in V_{i} \forall t \in T
\end{array}
$$

The objective of the aggregator is to minimize the charging cost in (3) subject to the state of charge (SOC) limit constraint (4), the EV charging energy limit constraint (5) and the charging energy non-negativity constraint (6). In (3), $A_{t}$ is the vector of the spot prices at time period $t$. The $n t h$ element of $A_{t}$ is the spot price $\alpha_{t}$ of the bus $n$ at time period $t . B_{t}$ is the matrix of the price sensitivity coefficients at time period $t$. It is a matrix with the price sensitivity coefficient $\beta_{t}$ of the bus $n$ at time period $t$ on the $n^{\text {th }}$ diagonal element. It is an aggregated form of (2). In the SOC limit constraint (4), $d_{j, t}$ indicates the driving energy consumption of the EV at time period $t$. It is corresponding to the expected driving distance of the EV at the time period $t$. The EV SOC level is calculated with the cumulated charging energy and driving energy consumption. In each time interval, the SOC levels of the EV battery are within the specified range. For the charging energy limit constraint (5), the EV charging energy is constrained by the maximum power limit and the expected EV charging availability. Parameter $p_{+}$is the maximum charging power. The EV charging availability parameter $s_{j, t}$ shows the expected status of the EV at time period $t$. Vehicle-to-grid (V2G) is not considered in this paper and EVs are regarded as demands. Therefore, the charging energy non-negativity constraint (6) holds. For the EV aggregators with regular customers of passenger cars, the best chance to schedule the charging plan is during the night when the EVs are parked at the original parking lot. For simplicity, it is assumed that the available periods for the EV charging scheduling of the aggregator is the period before the departure of the EV's first trip of the day and the period after the arrival of the EV's last trip of the day, as indicated in (7).

$$
s_{j, t}=\left\{\begin{array}{cc}
1 & t<t_{j}^{\text {dep }} \text { or } t>t_{j}^{\text {arr }} \\
0 & \text { otherwise. }
\end{array}\right.
$$

As shown in (4) and (5), the EV charging is constrained by the expected driving patterns of the EVs for the next day which are represented by the parameter $d_{j, t}$ and $s_{j, t}$. In the deterministic model, it is assumed that the aggregators have perfect information of EV driving patterns for the next day. The aggregators need to precisely predict the driving requirement and the charging availability of the EVs before they carry out the energy planning and submit the bids to the day-ahead electricity market. However, due to the randomness of the driving need, it is very difficult to perfectly predict the driving requirement and the charging availability of the EVs one day in advance. As a result, the energy planning with a deterministic model might not meet the actual driving requirements of the EVs. Because of the inherent randomness of the driving requirements, there is a chance that the EV user needs to drive for a longer distance or start the trips earlier than expected. In such cases, the energy plan may not be able to fulfill the actual driving need as the EV needs more energy or its available period for charging is shorter. Therefore, the optimal energy planning for the EV charging based on a deterministic approach cannot be actually realized by the aggregators.

Chance constrained programming is a direct and efficient tool to handle such a predicament. The randomness of the driving patterns lies in constraint (4) and (5). The driving distance $d_{j, t}$ and the charging availability $s_{j, t}$ are stochastic parameters dependent on the driving pattern of the EV. The two constraints can be reformulated in a chance constrained framework as,

$$
\operatorname{Pr}\left\{\begin{array}{c}
e_{j}^{-} \leq \sum_{t^{\prime}=t_{0}}^{t}\left(x_{j, t^{\prime}}-d_{j, t^{\prime}}\right)+e_{j, 0} \\
\sum_{t^{\prime}=t_{0}}^{t}\left(x_{j, t^{\prime}}-d_{j, t^{\prime}}\right)+e_{j, 0} \leq e_{j}^{+} \\
x_{j, t} \leq p_{+} s_{j, t}
\end{array} \forall t \in T\right\} \geq 1-\epsilon
$$

The chance constrained model of the optimal charging of aggregator $i$ is formed by objective (3) subject to the charging energy non-negativity constraint (6) and the probabilistic constraint (8). The probabilistic constraint (8) guarantees that the failure probability of the charging meeting the driving requirements is below the predetermined confidence parameter $\epsilon$ for each $\mathrm{EV}$.

As neither of the stochastic parameters $d_{j, t}$ nor $s_{j, t}$ in the chance constrained model follows a Gaussian distribution and their elements with different time index are correlated with each other, such a chance constrained optimization problem is hard to solve [29]. Notice that (a) the constraints are linear and the stochastic parameters are not the multipliers of the variables $x_{j, t}$, (b) the stochastic parameters have finite distribution and (c) the domain of the optimization is bounded, the chance constrained optimization can be solved by a mixedinteger programming method proposed by [29], [30]. A realization of the possible driving pattern is noted by the parameters $d_{j, k, t}$ and $s_{j, k, t}$ associated with the probability $\pi_{j, k}$. A binary variable $z_{j, k}$ is introduced for each driving pattern 
realization and the probabilistic constraint (8) can be reformulated as (9) to (12).

$$
\begin{gathered}
e_{j}^{-} \leq \sum_{t^{\prime}=t_{0}}^{t}\left(x_{j, t^{\prime}}-d_{j, k, t^{\prime}}\right)+e_{j, 0}+z_{j, k} \sum_{t^{\prime}=t_{0}}^{t} d_{j, k, t^{\prime}} \\
\forall j \in V_{i} \forall k \in R_{j} \forall t \in T \text { (9) } \\
\sum_{t^{\prime}=t_{0}}^{t}\left(x_{j, t^{\prime}}-d_{j, k, t^{\prime}}\right)+e_{j, 0} \leq e_{j}^{+}+z_{j, k} \sum_{t^{\prime}=t_{0}}^{t}\left(p_{+}-d_{j, k, t^{\prime}}\right) \\
\forall j \in V_{i} \forall k \in R_{j} \forall t \in T(10) \\
x_{j, t} \leq p_{+} s_{j, k, t}+p_{+} z_{j, k} \quad \forall j \in V_{i} \forall k \in R_{j} \forall t \in T \text { (11) } \\
\sum_{k \in R_{j}}\left(\pi_{j, k} z_{j, k}\right) \leq \epsilon \quad z_{j, k} \in\{0,1\} \quad \forall j \in V_{i}
\end{gathered}
$$

When the binary variable $z_{j, k}=0$, constraints (9) to (11) have a similar form as (4)-(5) and the constraints are guaranteed for the driving pattern realization $k$. When the binary variable $z_{j, k}=1$, constraints (9) to (11) are changed to (13) to (15).

$$
\begin{aligned}
e_{j}^{-} \leq \sum_{t^{\prime}=t_{0}}^{t} x_{j, t^{\prime}}+ & e_{j, 0} \\
& \forall j \in V_{i} \forall k \in R_{j} \forall t \in T \text { (13) } \\
\sum_{t^{\prime}=t_{0}}^{t}\left(x_{j, t^{\prime}}-p_{+}\right)+ & e_{j, 0} \leq e_{j}^{+} \\
\forall j \in V_{i} \forall k \in R_{j} \forall t \in T \text { (14) } & \begin{array}{r}
\forall j \\
x_{j, t} \leq p_{+}\left(s_{j, k, t}+1\right) \quad \forall j \in V_{i} \forall k \in R_{j} \forall t \in T \text { (15) }
\end{array}
\end{aligned}
$$

Constraints (13) to (15) are always satisfied in the domain of the optimization problem $\left(0 \leq x_{j, t} \leq p_{+}\right)$given a reasonable initial SOC condition that the SOC level of the EV battery is within limit $\left(e_{j}^{-} \leq e_{j, 0} \leq e_{j}^{+}\right)$. Therefore, the constraints of the driving pattern realization $k$ will not affect the solution of the optimization when $z_{j, k}=1$. In constraint (12), $\pi_{j, k}$ is the probability of the realization $k$ of the possible driving patterns. The knapsack constraint (12) is equivalent to the probabilistic constraint as shown in (16) and therefore the original probabilistic constraint (8) is satisfied.

$$
\sum_{k \in R_{j}} \pi_{j, k}\left(1-z_{j, k}\right) \geq 1-\epsilon z_{j, k} \in\{0,1\} \forall j \in V_{i} \text { (16) }
$$

For the probability of each possible driving pattern realization, $\pi_{j, k} \subset[0,1]\left(\forall k \in R_{j}\right)$. Therefore, the binary variable for the realization $k$ of the possible driving patterns $z_{j, k}=0$ when $\pi_{j, k}>\epsilon$, otherwise constraint (12) cannot be satisfied. Then the set of the possible driving patterns realizations $N_{R_{j}}$ can be divided into two subsets: the subset of possible driving pattern realizations with a probability over confidence parameter $R_{j+}=\left\{k \in R_{j}: \pi_{k}>\epsilon\right\}$ and the subset of possible driving pattern realizations with a probability less than or equal to confidence parameter $R_{j-}=\left\{k \in R_{j}: \pi_{k} \leq \epsilon\right\}$. In order to tighten the constraints, (9) to (12) can be formulated as (17) to (23) and the number of the binary variables is reduced.

$$
\begin{array}{r}
e_{j}^{-} \leq \sum_{t^{\prime}=t_{0}}^{t}\left(x_{j, t^{\prime}}-d_{j, k_{1}, t^{\prime}}\right)+e_{j, 0} \\
\forall j \in V_{i} \forall k_{1} \in R_{j+} \forall t \in T(17) \\
\sum_{t^{\prime}=t_{0}}^{t}\left(x_{j, t^{\prime}}-d_{j, k_{1}, t^{\prime}}\right)+e_{j, 0} \leq e_{j}^{+} \\
\forall j \in V_{i} \forall k_{1} \in R_{j+} \forall t \in T(18) \\
x_{j, t} \leq p_{+} s_{j, k_{1}, t} \quad \forall j \in V_{i} \forall k_{1} \in R_{j+} \forall t \in T(19) \\
\sum_{t^{\prime}=t_{0}}^{t}\left(x_{j, t^{\prime}}-d_{j, k_{2}, t^{\prime}}\right)+e_{j, 0} \leq e_{j}^{+}+z_{j, k_{2}} \sum_{t^{\prime}=t_{0}}^{t}\left(p_{+}-d_{j, k_{2}, t^{\prime}}\right) \\
\forall j \in V_{i} \forall k_{2} \in R_{j-} \forall t \in T(21)
\end{array}
$$

$$
\begin{gathered}
x_{j, t} \leq p_{+} s_{j, k_{2}, t}+p_{+} z_{j, k_{2}} \quad \forall j \in V_{i} \forall k_{2} \in R_{j-} \forall t \in T \\
\sum_{k_{2} \in R_{j-}}\left(\pi_{j, k_{2}} z_{j, k_{2}}\right) \leq \epsilon \quad z_{j, k_{2}} \in\{0,1\} \quad \forall j \in V_{i}
\end{gathered}
$$

For constraint (17) to (19), the driving pattern realization $k_{1}$ is met by the charging plan. For constraint (20) to (22), the driving pattern realization $k_{2}$ is met when the binary variable $z_{j, k_{2}}=0$. The constraints are always satisfied in the domain of the optimization when $z_{j, k_{2}}=1$. Constraint (23) guarantees that the failure probability of the charging plan is below the confidence parameter $\epsilon$ the same as constraint (8). The chance constrained programming model of aggregator $i$ is defined by the objective (3) subject to constraints (6) and (17) to (23).

\section{CAlCUlation OF DLMPs Through MIQP}

The concepts of DLMPs and DTs were proposed in [11], [18] and [19] to alleviate congestion in a distribution network in a decentralized manner. The DSO predicts the conventional demand in the distribution network and the spot prices at the relevant transmission buses. Accordingly, the DSO calculates the DLMPs based on the flexible demand data and conventional demand by the optimization respecting the network constraints. The calculated DTs are broadcasted to all the aggregators. The aggregators carry out their own optimal energy planning with the DTs and the predicted spot prices and submit their bids to the day-ahead market. The DLMP based approach to alleviate congestion due to EV demand in distribution system is illustrated in Fig. 1.

The objective of the DSO optimization is to minimize the total cost of electricity consumption in the distribution system in (24) subject to line flow constraint (25), the probabilistic constraint of the driving patterns (26) to (32) and the charging energy non-negativity constraint (33). The DC optimal power flow (DC OPF) is used for calculating DLMPs and DTs. The DC OPF has been widely used for LMP calculation in market operation and settlements due to good accuracy and high computation efficiency. It is considered sufficient in many cases, especially LMP calculation [31]. In industry, it has been employed by several software tools for chronological LMP simulation and forecasting [32]. For calculating DLMPs of distribution systems, the DC OPF is a good option considering the large number of nodes in distribution systems.

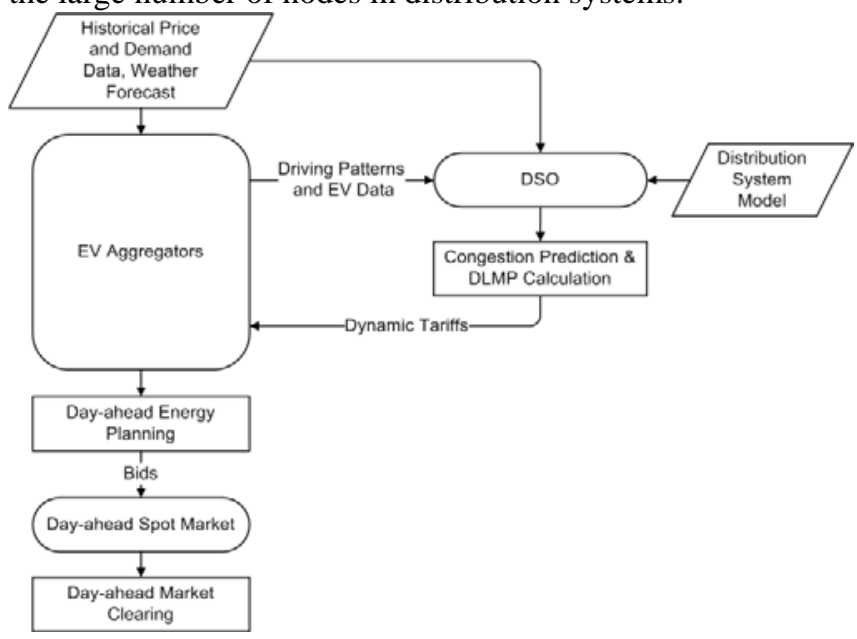

Fig. 1. DLMP based congestion management in the distribution system. 


$$
\begin{aligned}
\min _{x_{j, t}} & \sum_{j \in V} \sum_{t \in T}\left[\frac{1}{2}\left(E_{j} x_{j, t}\right)^{T} B_{t}\left(E_{j} x_{j, t}\right)+A_{t}^{T} E_{j} x_{j, t}\right] \\
& +\sum_{t \in T} A_{t}^{T} Q_{t}
\end{aligned}
$$

subject to

$$
\begin{gathered}
-f_{l} \leq \sum_{j \in V}\left(D_{l}^{T} E_{j} x_{j, t}\right)+D_{l}^{T} Q_{t} \leq f_{l} \\
\forall l \in L \forall t \in T \text { (25) } \\
e_{j}^{-} \leq \sum_{t^{\prime}=t_{0}}^{t}\left(x_{j, t^{\prime}}-d_{j, k_{1}, t^{\prime}}\right)+e_{j, 0} \\
\forall j \in V \quad \forall k_{1} \in R_{j+} \forall t \in T \text { (26) } \\
\sum_{t^{\prime}=t_{0}}^{t}\left(x_{j, t^{\prime}}-d_{j, k_{1}, t^{\prime}}\right)+e_{j, 0} \leq e_{j}^{+} \\
\forall j \in V \forall k_{1} \in R_{j+} \forall t \in T \text { (27) } \\
x_{j, t} \leq p_{+} s_{j, k_{1}, t} \quad \forall j \in V \forall k_{1} \in R_{j+} \forall t \in T \text { (28) } \\
e_{j}^{-} \leq \sum_{t^{\prime}=t_{0}}^{t}\left(x_{j, t^{\prime}}-d_{j, k_{2}, t^{\prime}}\right)+e_{j, 0}+z_{j, k_{2}} \sum_{t^{\prime}=t_{0}}^{t} d_{j, k_{2}, t^{\prime}} \\
\forall j \in V \forall k_{2} \in R_{j-} \forall t \in T(29) \\
\sum_{t^{\prime}=t_{0}}^{t}\left(x_{j, t^{\prime}}-d_{j, k_{2}, t^{\prime}}\right)+e_{j, 0} \leq e_{j}^{+}+z_{j, k_{2}} \sum_{t^{\prime}=t_{0}}^{t}\left(p_{+}-d_{j, k_{2}, t^{\prime}}\right) \\
\forall j \in V \forall k_{2} \in R_{j-} \forall t \in T(30) \\
x_{j, t} \leq p_{+} s_{j, k_{2}, t}+p_{+} z_{j, k_{2}} \quad \forall j \in V \forall k_{2} \in R_{j-} \forall t \in T \text { (31) } \\
\sum_{k_{2} \in R_{j-}}\left(\pi_{j, k_{2}} z_{j, k_{2}}\right) \leq \epsilon \quad z_{j, k_{2}} \in\{0,1\} \quad \forall j \in V(32) \\
x_{j, t} \geq 0 \quad \forall j \in V \quad \forall t \in T \text { (33) }
\end{gathered}
$$

The DLMPs of the buses in the distribution network can be obtained from the results of the DSO's optimization as,

$$
D L M P_{t}=A_{t}+\sum_{l \in N_{L}}\left[\left(\lambda_{l, t}^{+}-\lambda_{l, t}^{-}\right) D_{l}\right] \quad \forall t \in T \text { (34) }
$$

where $\lambda_{l, t}^{+}$and $\lambda_{l, t}^{-}$are the dual variables of the positive and negative flow constraints in (25). Accordingly, the DSO calculates the DTs $\rho_{t}$ defined by (35) and broadcasts them to the aggregators. The predicted spot prices and the price sensitivity coefficients used by the DSO are shared with the aggregators.

$$
\rho_{t}=D L M P_{t}-A_{t}=\sum_{l \in N_{L}}\left[\left(\lambda_{l, t}^{+}-\lambda_{l, t}^{-}\right) D_{l}\right] \quad \forall t \in T \text { (35) }
$$

With the DTs, the aggregators carry out their own optimal energy planning of the EV charging. For aggregator $i$, the objective of its optimization is to minimize the charging cost in (36) subject to the charging energy non-negativity constraint (6) and the probabilistic constraint of the driving patterns (17) to (23). The electricity prices for the aggregators consist of the DTs and the spot prices. The DTs are the marginal value of the network constraints in the DSO's optimization and consequently the network constraints are respected by the aggregators' optimizations. The convergence of the results of the DSO and the aggregators' solutions has been proved in [11], [19]. The binary variables in the chance constraints are determined according to the driving pattern realizations. The binary variables of the driving pattern realizations with a long daily driving distance and a short charging period will equal to 1 within the limit of the knapsack constraint (23) and (32) in both the DSO and the aggregators' models. The consistency of the binary variables can be guaranteed by the DSO. When the binary variables are fixed, the DSO and the aggregators' optimizations will converge as in the standard DLMP framework.

$$
\min _{x_{j, t}} \sum_{j \in V_{i}} \sum_{t \in T}\left[\frac{1}{2}\left(E_{j} x_{j, t}\right)^{T} B_{t}\left(E_{j} x_{j, t}\right)+\left(A_{t}+\rho_{t}\right)^{T} E_{j} x_{j, t}\right]
$$

As presented above, both the chance constrained optimizations of the DSO and the aggregators are formulated through MIQP, which can be solved by a number of commercial solvers [33]. The distributions of the EV driving patterns used in the optimization of the DSO and the aggregators should have the same stochastic characteristics of the driving behaviors, which can be obtained from either thirdparty statistical surveys or historical data of the EV charging.

\section{CASE STUdies}

In order to illustrate the efficacy of the proposed DLMP approach through chance constrained MIP, case studies were conducted with a 33-bus distribution system of the Bornholm power system using the Danish driving pattern data.

Three scenarios were selected for the case studies as follows:

Case 1: Case 1 is the base case which shows the situation when there is no congestion management. There is no pricing signal from the DSO to the aggregators. The aggregators carry out their energy planning only with the predicted spot price of electricity.

Case 2: In Case 2, the proposed chance constrained models are applied in both the DSO and the aggregators' optimization. It shows the case when the proposed approach is applied.

Case 3: In Case 3, the DSO carries out its optimization with the deterministic model. In order to maintain the satisfaction of the customers and guarantee the driving needs for the next day are met with a certain confidence level, the aggregators use the chance constrained model. This case shows the possible failure of congestion management if the DSO takes a deterministic model to calculate the DLMPs while the aggregators need to handle the uncertainty of the EV driving patterns for the next day.

The details of the case studies are presented in the following subsections.

\section{A. Grid Data}

The single line diagram of the 33-bus distribution system is shown in Fig. 2.

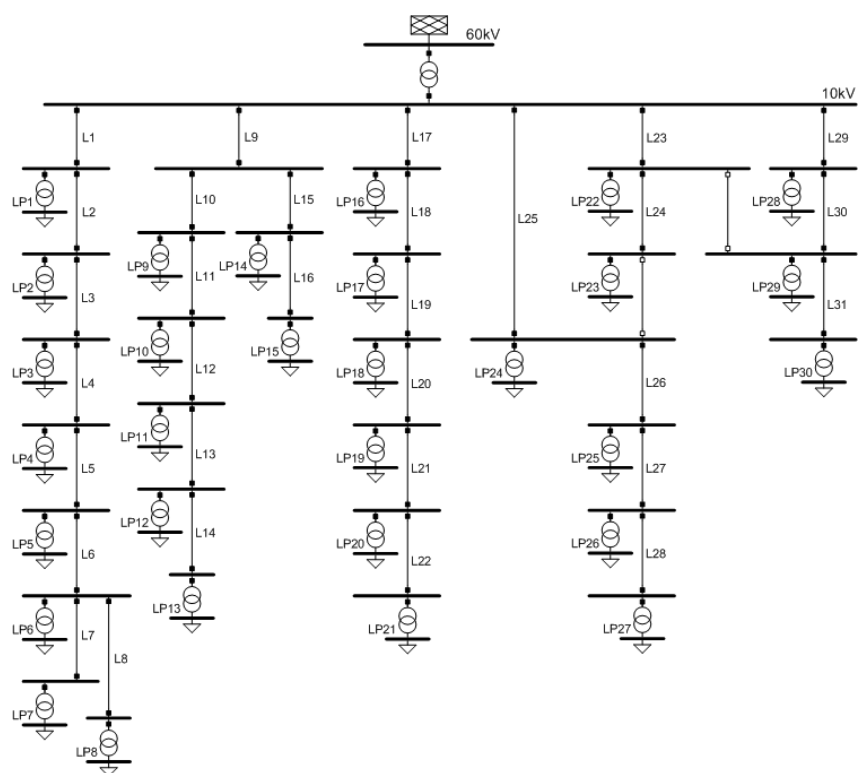

Fig. 2. Single line diagram of the distribution system for the case studies. 
In the distribution system, the $400 \mathrm{~V}$ customers in 30 load points (LPs) are connected to the $10 \mathrm{kV}$ distribution network through the secondary transformers in 6 feeders. The $10 \mathrm{kV}$ network is connected to the $60 \mathrm{kV}$ bus through a 10 MVA transformer. The $60 \mathrm{kV}$ bus, which is the connection point of the distribution system to the external grid, is set as the slack bus in the case studies.

Four types of cables are used in the distribution network for case studies. The loading limits of the lines are listed in Table I.

TABLE I

Line Loading Limit in the Distribution System

\begin{tabular}{lc}
\hline \multicolumn{1}{c}{ Lines } & Limit $(\mathrm{kW})$ \\
\hline L7 & 1657 \\
\hline L2-L6, L8, L10-L16, L18-L21, L24, L26-L30 & 2132 \\
\hline L22, L23, L31 & 2510 \\
\hline L9, L17, L25 & 2936 \\
\hline
\end{tabular}

The EV penetration level in the case studies is set as $100 \%$. $100 \%$ EV penetration means that all the private passenger cars are EVs in the distribution network. Two EV aggregators are assumed in the case studies. One aggregator has contracts with $40 \%$ of the EVs on each load point while the other has contracts with the rest $60 \%$ of EVs on the LPs. The EV numbers contracted with the aggregators and the conventional demand data of the LPs in the distribution system are listed in Table II. The total EV demand is about $18 \%$ of the load on the LPs in the distribution network on average.

TABLE II

Data of the Load in the Distribution System

\begin{tabular}{|c|c|c|c|c|c|c|}
\hline \multirow{3}{*}{ LPs } & \multicolumn{4}{|c|}{ Conventional Demand } & \multicolumn{2}{|c|}{ EV No. } \\
\hline & \multicolumn{2}{|c|}{ Residential Load } & \multicolumn{2}{|c|}{ Non-Residential Load } & \multirow{2}{*}{$\begin{array}{c}\text { Aggr } \\
\text { egato } \\
\text { r } 1\end{array}$} & \multirow{2}{*}{$\begin{array}{c}\text { Aggr } \\
\text { egato } \\
\text { r } 2\end{array}$} \\
\hline & $\begin{array}{l}\text { Ave. } \\
(\mathrm{kW})\end{array}$ & $\begin{array}{l}\text { Peak } \\
(\mathrm{kW})\end{array}$ & $\begin{array}{l}\text { Ave. } \\
(\mathrm{kW})\end{array}$ & $\begin{array}{l}\text { Peak } \\
(\mathrm{kW})\end{array}$ & & \\
\hline LP1 & 37.1 & 22.7 & 14.1 & 11.5 & 13 & 20 \\
\hline LP2 & 154.9 & 94.6 & 20.1 & 16.3 & 55 & 83 \\
\hline LP3 & 26.9 & 16.4 & 12.8 & 10.4 & 9 & 15 \\
\hline LP4 & 114.6 & 70.0 & 44.3 & 35.9 & 40 & 62 \\
\hline LP5 & 77.2 & 47.1 & 9.2 & 7.4 & 27 & 42 \\
\hline LP6 & 105.8 & 64.6 & 45.9 & 37.2 & 37 & 57 \\
\hline LP7 & 42.9 & 26.2 & 9.0 & 7.3 & 15 & 23 \\
\hline LP8 & 5.6 & 3.4 & 2.1 & 1.7 & 2 & 3 \\
\hline LP9 & 87.3 & 53.3 & 33.2 & 26.9 & 31 & 47 \\
\hline LP10 & 69.9 & 42.7 & 4.1 & 3.3 & 24 & 38 \\
\hline LP11 & 70.2 & 42.9 & 0.5 & 0.4 & 25 & 38 \\
\hline LP12 & 150.3 & 91.8 & 6.5 & 5.2 & 53 & 81 \\
\hline LP13 & 36.5 & 22.3 & 25.7 & 20.8 & 13 & 20 \\
\hline LP14 & 103.1 & 62.9 & 7.9 & 6.4 & 36 & 56 \\
\hline LP15 & 181.5 & 110.8 & 21.6 & 17.5 & 64 & 98 \\
\hline LP16 & 45.4 & 27.7 & 63.1 & 51.1 & 16 & 24 \\
\hline LP17 & 55.4 & 33.8 & 28.8 & 23.3 & 19 & 30 \\
\hline LP18 & 5.0 & 3.1 & 1.9 & 1.5 & 1 & 3 \\
\hline LP19 & 43.5 & 26.5 & 72.2 & 58.5 & 15 & 24 \\
\hline LP20 & 0.6 & 0.4 & 0.2 & 0.2 & 0 & 1 \\
\hline LP21 & 64.6 & 39.5 & 31.8 & 25.8 & 23 & 35 \\
\hline LP22 & 723.0 & 441.4 & 244.2 & 197.8 & 257 & 387 \\
\hline LP23 & 223.5 & 136.5 & 85.1 & 69.0 & 79 & 120 \\
\hline
\end{tabular}

\begin{tabular}{lllllll}
\hline LP24 & 226.6 & 138.4 & 86.3 & 69.9 & 80 & 122 \\
\hline LP25 & 184.4 & 112.6 & 77.0 & 62.4 & 65 & 99 \\
\hline LP26 & 121.9 & 74.4 & 108.5 & 87.9 & 43 & 66 \\
\hline LP27 & 34.2 & 20.9 & 76.3 & 61.8 & 12 & 19 \\
\hline LP28 & 55.4 & 33.8 & 14.6 & 11.8 & 19 & 30 \\
\hline LP29 & 99.4 & 60.7 & 43.7 & 35.4 & 35 & 54 \\
\hline LP30 & 111.5 & 68.0 & 34.8 & 28.2 & 39 & 60 \\
\hline
\end{tabular}

\section{B. EV and Driving Pattern Data}

The key parameters of the EVs are listed in Table III.

TABLE III

EV Data Profile for Case Studies

\begin{tabular}{ll}
\hline \multicolumn{1}{c}{ Parameter } & \multicolumn{1}{c}{ Value } \\
\hline EV battery capacity & $60 \mathrm{kWh}$ \\
\hline Charging power limit & $10 \mathrm{~kW}(3-\mathrm{phase})$ \\
\hline Energy consumption per $\mathrm{km}$ & $150 \mathrm{Wh} / \mathrm{km}$ \\
\hline Lower SOC level limit & $20 \%$ \\
\hline Upper SOC level limit & $85 \%$ \\
\hline
\end{tabular}

The driving pattern data used in the case studies are obtained from a dataset of real driving data on weekdays obtained from the Danish National Travel Survey. The distribution of the driving patterns is shown in Fig. 3 and Fig. 4.

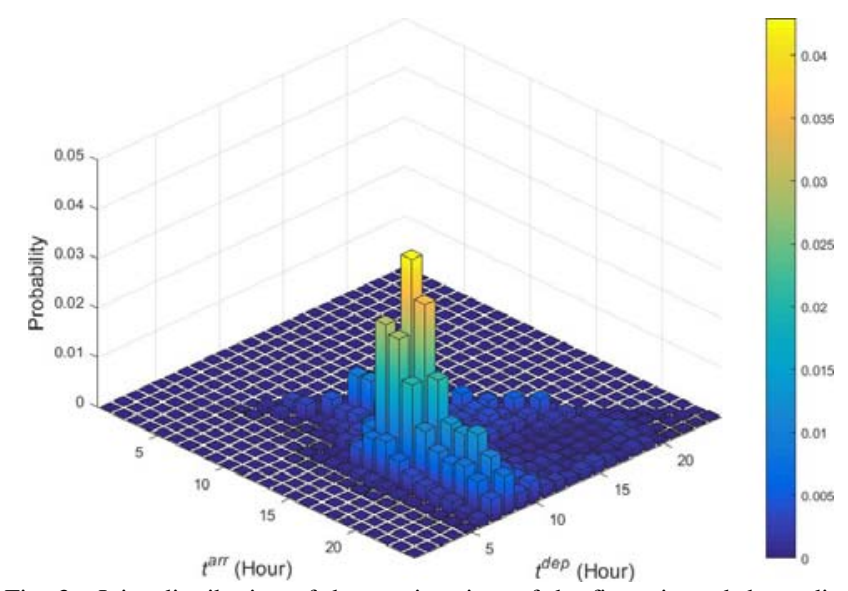

Fig. 3. Joint distribution of the starting time of the first trip and the ending time of the last trip.

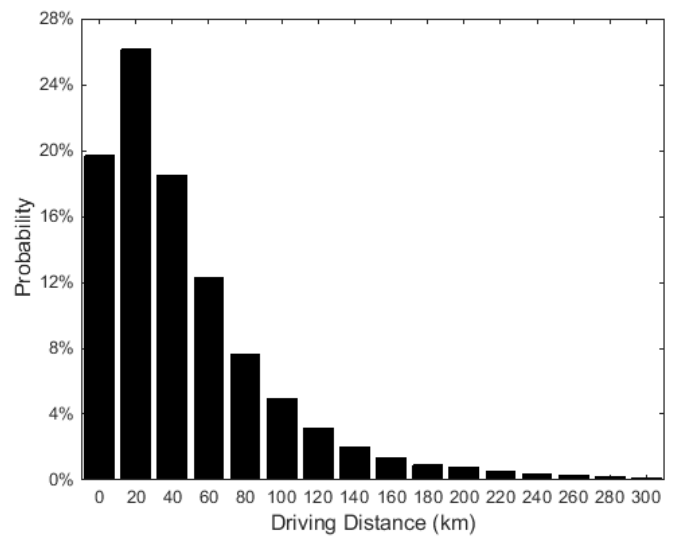

Fig. 4. Daily driving distance distribution. 
The daily driving distance and the starting and ending time of the driving patterns are coupled as a 3-dimension random variable. For each driving pattern realization, it is with the daily driving distance, the starting and ending time as well as the probability of the realization. The starting and ending time are interpreted to the charging availability according to (7). Without losing generality, the driving distance of the driving pattern realization is assumed to increase linearly from 0 to the daily driving distance from the starting time to the ending time of the driving pattern. 10 different driving pattern realizations with its own probability as listed in Table IV are generated from the joint distribution. The charging shall guarantee the driving pattern realizations with the confidence defined in the probabilistic constraints for all the EVs are met. The confidence parameter in the chance constrained models is set as $5 \%$ in the case studies.

TABLE IV

Driving Pattern Realizations in the Chance Constrained Models

\begin{tabular}{lcccc}
\hline No. & $\begin{array}{c}\text { Starting } \\
\text { time }\end{array}$ & $\begin{array}{c}\text { Ending } \\
\text { time }\end{array}$ & $\begin{array}{c}\text { Driving } \\
\text { Distance }\end{array}$ & Probability \\
\hline 1 & 4 & 23 & $140 \mathrm{~km}$ & 0.00169 \\
\hline 2 & 4 & 24 & $140 \mathrm{~km}$ & 0.00003 \\
\hline 3 & 5 & 23 & $140 \mathrm{~km}$ & 0.71116 \\
\hline 4 & 5 & 24 & $140 \mathrm{~km}$ & 0.03383 \\
\hline 5 & 4 & 23 & $150 \mathrm{~km}$ & 0.00008 \\
\hline 6 & 5 & 23 & $150 \mathrm{~km}$ & 0.00671 \\
\hline 7 & 5 & 24 & $150 \mathrm{~km}$ & 0.00049 \\
\hline 8 & 4 & 23 & $160 \mathrm{~km}$ & 0.00032 \\
\hline 9 & 5 & 23 & $160 \mathrm{~km}$ & 0.04367 \\
\hline 10 & 5 & 24 & $160 \mathrm{~km}$ & 0.00506 \\
\hline
\end{tabular}

In the proposed model, the number of the binary variables increases with the number of the EVs. In order to save the time and memory consumption of the calculation, the EVs are grouped by a factor of 10 and scaled up in the optimizations of the case studies.

\section{Case Study Results}

The case studies were carried out with the General Algebraic Modeling System (GAMS) optimization software using the commercial solver CPLEX [34].

1) Case 1: In the case without any congestion management, the aggregators carry out their own energy planning to minimize the EV charging cost given the spot prices, respectively. The spot prices used in the case studies are shown in Fig. 5. In the case studies, Line L23 has the highest loading level among the lines in the 33-bus distribution system. The loading of L23 without DLMP is shown in Fig. 6.

If the DLMP is not applied, congestion due to the EV charging demand occurs at 3:00 am when the spot price is the lowest. All the aggregators tend to charge the EVs at that time for the lowest charging cost and it results in a high charging demand.

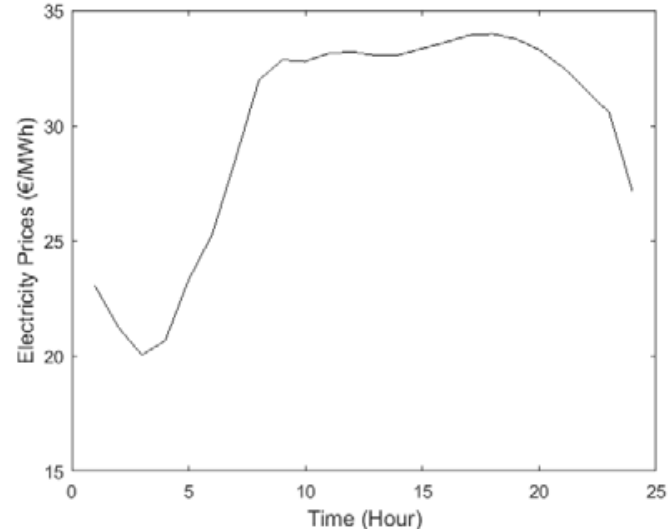

Fig. 5. Spot prices used in the case studies.

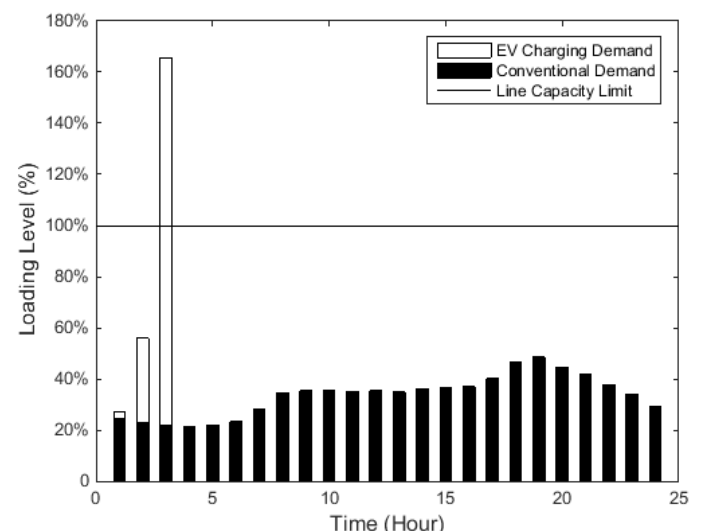

Fig. 6. Loading level of Line L23 without DLMP.

2) Case 2: In order to alleviate the congestion due to the EV charging shown in Fig. 6, the proposed DLMP approach through chance constrained MIP for congestion management was implemented in Case 2. The DLMPs and DTs of the nodes in the distribution system were calculated by the DSO optimization through the chance constrained programming. The calculated DLMPs and the spot prices of the electricity are shown in Fig. 7. The DLMPs and DTs of some heavyloaded LPs around the peak hours are listed in Table V.

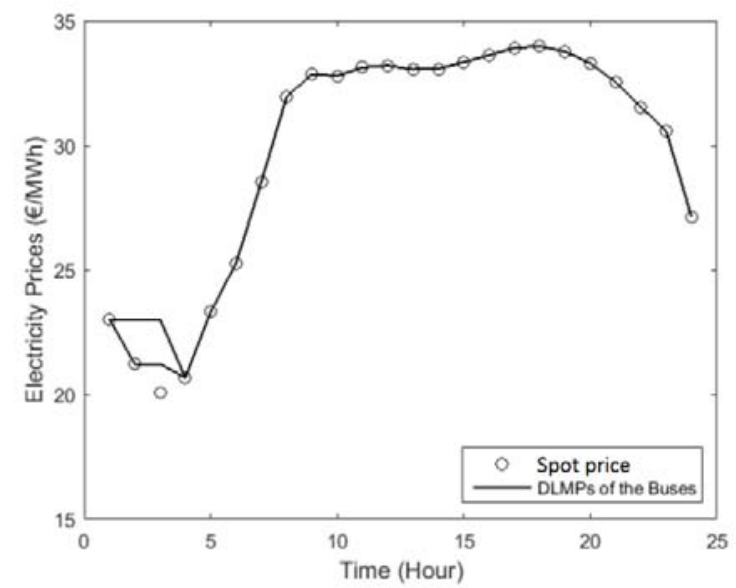

Fig. 7. DLMPs calculated through chance constrained programming. 
TABLE V

DLMPs and DTs (€/MWh) around Peak Hours Calculated through Chance Constrained Programming

\begin{tabular}{lcccc}
\hline Time (Hour) & 1 & 2 & 3 & 4 \\
\hline Spot Price & 23.01 & 21.23 & 20.05 & 20.68 \\
\hline DLMPs on LP22 & 23.01 & 23.01 & 23.01 & 20.68 \\
\hline DTs on LP22 & 0 & 1.78 & 2.96 & 0 \\
\hline DLMPs on LP23 & 23.01 & 23.01 & 23.01 & 20.68 \\
\hline DTs on LP23 & 0 & 1.78 & 2.96 & 0 \\
\hline DLMPs on LP24 & 23.01 & 21.23 & 21.23 & 20.68 \\
\hline DTs on LP24 & 0 & 0 & 1.18 & 0 \\
\hline
\end{tabular}

As shown in Fig. 7 and Table V, the positive DTs appear at 2:00 and 3:00 in the morning when the congestion is expected to happen due to the EV charging demand at the corresponding nodes in the distribution network. It is the period when the EV charging demand is high in Case 1. The positive DTs increase the electricity pricing during the peak hours and motivate the aggregators to disperse the EV charging demand to a lighter loading period.

In the proposed DLMP framework, the DTs calculated by the DSO are sent to the aggregators and the aggregators carry out their own energy planning of the EV charging respectively. In order to verify the behaviors of the aggregators with the proposed DLMP approach through chance constrained programming, the aggregators' energy planning was simulated. The aggregators' energy planning for EV charging were carried out independently to minimize their own charging cost with the DTs and the predicted spot prices. The loading of Line L23 in Case 2 is shown in Fig. 8. As shown in the figure, the peak of the electricity load at 3:00 am in Case 1 is limited and the EV charging demand is shifted to the first two hours. Due to the positive DTs at 2:00 and 3:00 am, the costs for the charging at these two hours are increased. Consequently, the charging demands at these two hours are under the limit of the distribution network constraints and the congestion due to the EV charging demand is alleviated.

A Monte-Carlo simulation has been carried out to assess the satisfaction of the driving pattern chance constraint. The charging plans of the EVs are as the solutions of the aggregators' optimization. The real driving records in the original dataset from the Danish National Travel Survey are randomly assigned to the EVs to see if the charging plans with the chance constrained model satisfy the probabilistic constraint in the case. The simulation result shows that the violation probability of the charging for all the EVs of both aggregators in the case is within $4.99 \%$. Less than $5 \%$ of the EVs will have a driving pattern that the charging plans by the aggregators do not satisfied. Therefore, probabilistic constraint setting in the case studies is respected.

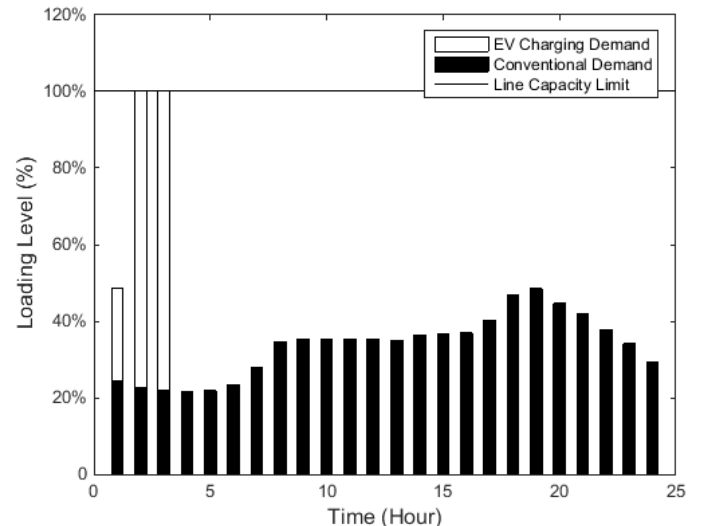

Fig. 8. Loading level of Line L23 with DLMP through chance constrained programming.

3) Case 3: In order to illustrate the ineffective results of the DLMP with deterministic modeling of the EV driving patterns, the case with DLMPs through deterministic optimization on the DSO side was simulated in Case 3. Because the EV aggregators need to maintain the customer satisfaction and it is difficult for the aggregators to perfectly predict the driving patterns of the EVs for the next day, the aggregators are assumed to perform the EV energy planning stochastically with the chance constrained model. Case 3 is to show that in this case, the congestion management will fail if the DSO uses a deterministic model to perform the DLMP method and calculate the DTs. Therefore, the EV aggregators are assumed to use the chance constrained optimization and the deterministic model is applied on the DSO side in case 3. The DLMPs and DTs of the nodes in the distribution system were calculated through the DSO optimization with the deterministic modeling of the EV driving patterns. The DLMPs and the spot prices of the electricity are shown in Fig. 9. The DLMPs and DTs of some heavy-loaded LPs in the distribution system around the peak hours are also listed in Table VI.

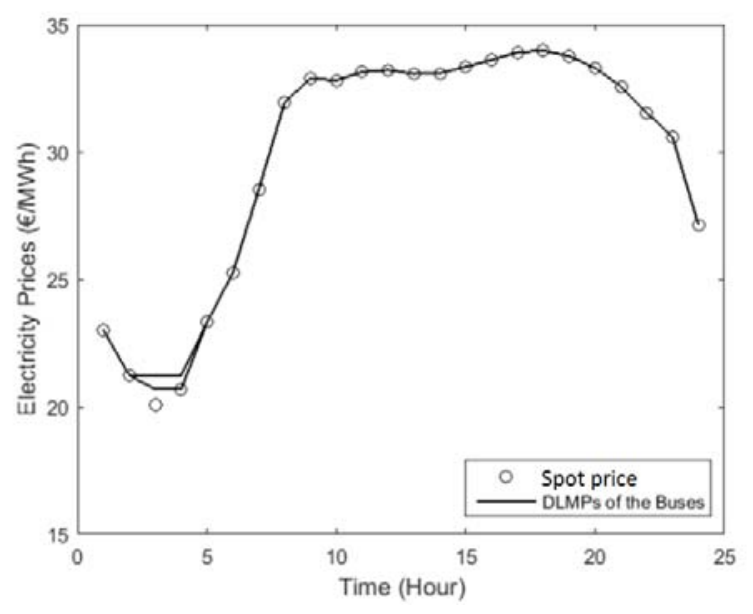

Fig. 9. DLMPs calculated with deterministic optimizations. 
TABLE VI

DLMPs and DTs (€/MWh) around Peak Hours Calculated with Deterministic Optimization

\begin{tabular}{lcccc}
\hline Time (Hour) & 1 & 2 & 3 & 4 \\
\hline Spot Price & 23.01 & 21.23 & 20.05 & 20.68 \\
\hline DLMPs on LP22 & 23.01 & 21.23 & 21.23 & 21.23 \\
\hline DTs on LP22 & 0 & 0 & 1.21 & 0.55 \\
\hline DLMPs on LP23 & 23.01 & 21.23 & 21.23 & 21.23 \\
\hline DTs on LP23 & 0 & 0 & 1.21 & 0.55 \\
\hline DLMPs on LP24 & 23.01 & 21.23 & 20.68 & 20.68 \\
\hline DTs on LP24 & 0 & 0 & 0.63 & 0 \\
\hline
\end{tabular}

As shown in Fig. 9 and Table VI, positive DTs appear at 3:00 and 4:00 in the morning. In the DSO optimization with a deterministic model, the EV charging demand is planned according to the obtained deterministic driving patterns. The stochastic features of the driving patterns are not taken into account. Therefore, the available period for the EV charging will be extended to the furthest limit even though there is a probability for the energy planning not to meet the EV driving requirements. Consequently, the EV charging is expected to take place at the hours with the lowest electricity prices in a larger available period. The calculated DTs may therefore differ from the case with chance constrained programming on the DSO side. In this case, positive DTs appear at 4:00 am while the DTs at 2:00 am remain zero. The DTs at 3:00 am are less than the DTs at the same period with the DLMP approach through the chance constrained programming.

Such differences of the DTs will result in different charging plans at the aggregator side. In order to illustrate the behaviors of the aggregators with DLMP through deterministic optimizations, the aggregators' energy planning was simulated in Case 3. The aggregators' optimizations were carried out independently with the DTs and the predicted spot energy prices. The optimizations through chance constrained programming were used at the aggregator side so that the EV driving requirements are guaranteed. The loading of Line L23 in Case 3 is shown in Fig. 10.

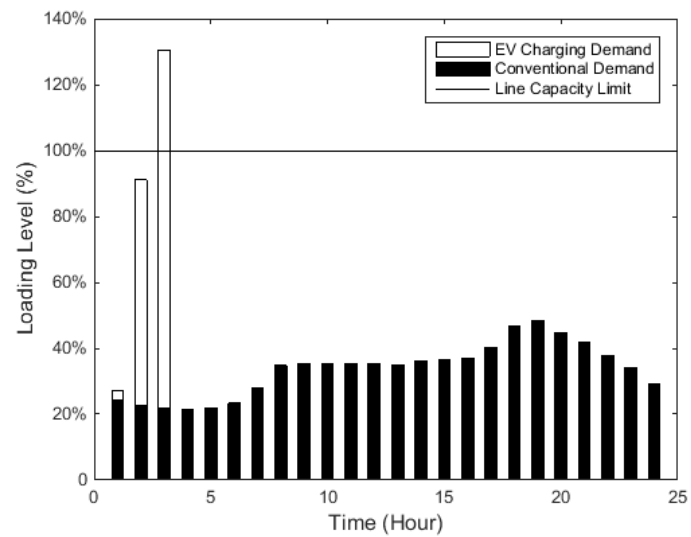

Fig. 10. Loading level of Line L23 with DLMP through deterministic optimization.
As shown in Fig. 10, the congestion happens on Line L23 at 3:00 am when the deterministic optimization is used on the DSO side. In the DSO optimization with a deterministic model of the EV driving patterns, the aggregators are expected to charge their EVs mainly from 3:00 to 4:00 in the morning. The calculated DTs are to balance the EV charging demand in the period. However, due to the stochastic characteristics of the driving patterns, the aggregators tend to charge the EVs in the first three hours in order to meet the driving requirements. As a result, the EV charging demand at 3:00 am is underestimated in the deterministic optimization on the DSO side. The congestion in Line L23 at 3:00 am is therefore not efficiently alleviated by the DTs. Such mis-estimations of the aggregators' behaviors in the deterministic optimization model will result in an ineffective outcome of the DLMP approach in handling the congestion in the distribution system due to the EV charging.

4) Cost analysis: The chance constrained approach is more conservative than a deterministic approach. It refers to the case when both the DSO and the aggregators do not consider the driving pattern uncertainty in their optimization model [11]. When the deterministic approach is used, the average cost for the EV charging is about 5\% lower than the case with the chance constrained models (from about 20.9EUR/MWh to 21.8EUR/MWh on average). The cost difference is not significant.

In the DLMP framework, the aggregator will pay the extra cost. Since the aggregator can include the uncertainties of the driving pattern in their EV scheduling optimization, it will feel more secure with the energy plan for EVs and will be willing to pay a bit more to have the security of the EV energy planning. For EV owners, it depends on the contracts between the EV owners and the EV aggregator. If the EV owner has to bear the extra cost, it should be reasonable to state the EV owner is willing to pay a bit more to have the flexibility to deviate a bit from the driving plan for the next day.

\section{CONCLUSIONS}

The DLMP has been shown to be efficient for the congestion management in the distribution networks with a high penetration level of EVs. However, the randomness of the driving requirements leads to difficulty in predicting the driving behavior precisely and therefore results in ineffective outcomes of the DLMP method with a deterministic model. Stochastic characteristics of the driving patterns are addressed in this paper by introducing probabilistic constraints in the DSO and aggregators' optimizations. It guarantees that the failure probability of the EV charging plans meeting the driving requirement is below the predetermined confidence parameter. The chance constrained optimizations of the DSO and the aggregators are formulated and solved through the MIQP. The case study results have demonstrated that with the DTs determined by the DSO through the chance constrained MIQP, aggregators taking into account the stochastic characteristics of the EV driving pattern plan their EV charging respecting the network constraints as expected and congestion in the distribution network is alleviated. 
The chance constrained approach is more conservative than a deterministic approach. With the chanced constrained DLMP, the aggregator will pay a bit more. However, the extra cost is about $5 \%$ and it is not significant. Therefore, the aggregator will be willing to pay a bit more to have the security of the EV energy planning. For the EV owners, they should be willing to pay a bit more to have the flexibility to deviate a bit from the driving plan for the next day due to the small cost difference.

For future work, the sensitivity of the chance constrained DLMP approach on the difference between the driving pattern distributions used by the DSO and the aggregators will be investigated. Further, the uncertainty also comes from the electricity price and demand forecast besides the EV driving patterns. An extended framework will be developed to include different sources of uncertainty. In addition, more practical issues in the power system including the line losses and the voltage constraints will also be studied.

\section{REFERENCES}

[1] C. C. Chan and Y. S. Wong, "Electric vehicles charge forward," IEEE Power Energy Mag., vol. 2, no. 6, pp. 24-33, 2004.

[2] J. C. Mukherjee and A. Gupta, "A Review of Charge Scheduling of Electric Vehicles in Smart Grid,” IEEE Syst. J., vol. PP, no. 99, pp. 113, 2014.

[3] S. Habib and M. Kamran, "A Novel Vehicle-to-Grid Technology with Constraint Analysis-A Review," in Proc. 2014 International Conference on Emerging Technologies (ICET), pp. 69-74.

[4] T. Trigg and P. Telleen. (2013, Apr.). Global EV Outlook. International Energy Agency, [Online]. Available: https:// www.iea.org/publications/globalevoutlook_2013.pdf

[5] J. T. Salihi, "Energy Requirements for Electric Cars and Their Impact on Electric Power Generation and Distribution Systems," IEEE Trans. Industry Application, vol. IA-9, no. 5, pp. 516-532, Apr. 1973.

[6] G. T. Heydt, "The impact of Electric Vehicle Deployment on Load Management Strategies," IEEE Trans. Power Apparatus and Systems, vol. PAS-102, no. 5, pp. 1253-1259, Apr. 1983.

[7] S. Rahman, G. B. Shrestha, "An investigation into the impact of electric vehicle load on the electric utility distribution system," IEEE Trans. Power Delivery, vol. 8, no. 2, pp. 591-597, Apr. 1993.

[8] J. A. P. Lopes, F. J. Soares, and P. M. R. Almeida, "Integration of Electric Vehicles in the Electric Power System," Proceedings of the IEEE, vol. 99, no. 1, pp. 168-183, Jan. 2011.

[9] K. Clement-Nyns, E. Haesen, and J. Driesen, "The Impact of Charging Plug-In Hybrid Electric Vehicles on a Residential Distribution Grid," IEEE Trans. Power Syst., vol. 25, no. 1, pp. 371-380, Feb. 2010.

[10] B. Biegel, P. Andersen, J. Stoustrup, and J. Bendtsen, "Congestion management in a smart grid via shadow prices," in Proc. 2012 8th IFAC Symposium on Power Plant and Power System Control, pp. 518-523.

[11] S. Huang, Q. Wu, S. S. Oren, R. Li, and Z. Liu, "Distribution locational marginal pricing through quadratic programming for congestion management in distribution networks," IEEE Trans. Power Syst., vol. PP, no. 99, pp. 1-9, Sep. 2014.

[12] R. Bohn, M. Caramanis, and F. Schweppe, "Optimal pricing in electrical networks over space and time," The RAND. J. Econ., vol. 15, no. 3, pp. 360-376, 1984.

[13] P. M. Sotkiewicz and J. M. Vignolo, "Nodal pricing for distribution networks: efficient pricing for efficiency enhancing DG," IEEE Trans. Power Syst., vol. 21, no. 2, pp. 1013-1014, May 2006.

[14] R. K. Singh and S. K. Goswami, "Optimum allocation of distributed generations based on nodal pricing for profit, loss reduction, and voltage improvement including voltage rise issue," Int. J. Electr. Power Energy Syst., vol. 32, no. 6, pp. 637-644, Jul. 2010.

[15] F. Meng and B. H. Chowdhury, "Distribution LMP-based economic operation for future smart grid," in Proc. 2011 IEEE Power and Energy Conference at Illinois, pp. 1-5.

[16] G. T. Heydt, B. H. Chowdhury, M. L. Crow, D. Haughton, B. D. Kiefer, F. Meng, and B. R. Sathyanarayana, "Pricing and control in the next generation power distribution system," IEEE Trans. Smart Grid, vol. 3, no. 2, pp. 907-914, Jun. 2012.

[17] K. Shaloudegi, N. Madinehi, S. H. Hosseinian, and H. A. Abyaneh, "A novel policy for locational marginal price calculation in distribution systems based on loss reduction allocation using game theory," IEEE Trans. Power Syst., vol. 27, no. 2, pp. 811-820, May 2012.

[18] N. O’Connell, Q. Wu, J. Østergaard, A. H. Nielsen, S. T. Cha, and Y. Ding, "Day-ahead tariffs for the alleviation of distribution grid congestion from electric vehicles," Electr. Power Syst. Res., vol. 92, pp. 106-114, Nov. 2012.

[19] R. Li, Q. Wu, and S. S. Oren, "Distribution locational marginal pricing for optimal electric vehicle charging management," IEEE Trans. Power Syst., vol. 29, no. 1, pp. 203-211, Jan. 2014.

[20] W. Liu, Q. Wu, F. Wen, and J. Østergaard, "Day-Ahead Congestion Management in Distribution Systems Through Household Demand Response and Distribution Congestion Prices," IEEE Trans. Smart Grid, vol. 5, no. 6, pp. 2739-2747, Jul. 2014.

[21] S. I. Vagropoulos and A. G. Bakirtzis, "Optimal bidding strategy for electric vehicle aggregators in electricity markets," IEEE Trans. Power Syst., vol. 28, no. 4, pp. 4031-4041, Nov. 2013.

[22] M. G. Vayá and G. Andersson, "Smart charging of plug-in vehicles under driving behaviour uncertainty," in Proc. Int. Conf. Probabilistic Methods Applied to Power Systems (PMAPS) 2012, pp. 1029-1034.

[23] M. G. Vayá and G. Andersson, "Optimal Bidding Strategy of a Plug-In Electric Vehicle Aggregator in Day-Ahead Electricity Markets Under Uncertainty," IEEE Trans. Power Syst., vol. PP, no. 99, pp. 1-11, Oct. 2014.

[24] A. Prékopa, "Probabilistic programming," Stochastic Programming in Handbooks in Operations Research and Management Science, vol. 10, pp. 267-351, 2003.

[25] C. M. Lagoa, X. Li, and M. Sznaier, "Probabilistically constrained linear programs and risk-adjusted controller design," SIAM Journal on Optimization, vol. 15, no. 3, pp. 938-951, 2005.

[26] R. Henrion, and C. Strugarek, "Convexity of chance constraints with independent random variables," Computational Optimization and Applications, vol. 41, no. 2, pp. 263-276, 2008.

[27] R. A. Verzijlbergh, Z. Lukszo, and M. D. Ilic, "Comparing different EV charging strategies in liberalized power systems," in Proc. 2012 9th International Conference on the European Energy Market, pp. 1-8.

[28] R. A. Verzijlbergh, L. J. De Vries, and Z. Lukszo, "Renewable energy sources and responsive demand. Do we need congestion management in the distribution grid?," IEEE Trans. Power Syst., vol. PP, no. 99, pp. 110, Feb. 2014.

[29] J. Luedtke, S. Ahmed, and G. L. Nemhauser, "An integer programming approach for linear programs with probabilistic constraints," Math. Program., vol. 122, pp. 247-272, Apr. 2010.

[30] A. Ruszczynski, "Probabilistic programming with discrete distributions and precedence constrained knapsack polyhedra," Math. Program., vol. 93, pp. 195-215, Dec. 2002.

[31] F. Li and R. Bo, "DCOPF-based LMP simulation: algorithm, comparison with ACOPF, and sensitivity," IEEE Trans. Power Syst., vol. 22, no. 4, pp. 1475-1485, Nov. 2007.

[32] J. Yang, F. Li, and L. A. A. Freeman, "A market simulation program for the standard market design and generation/transmission planning," in Proc. IEEE Power Energy Soc. General Meeting, 2003, pp. 442-446.

[33] GAMS Development Corporation (2014, Dec.). GAMS -- The Solver Manuals. GAMS Development Corporation, Washington DC, USA. [Online]. Available: http://www.gams.com/ dd/docs/solvers/allsolvers.pdf

[34] R. E. Rosenthal (2014, Dec.). GAMS -- A User’s Guide. GAMS Development Corporation, Washington DC, USA. [Online]. Available: http://www.gams.com/dd/docs/bigdocs/GAMSUsersGuide.pdf 